\title{
Circular RNAs in Cardiac Regeneration: Cardiac Cell Proliferation, Differentiation, Survival, and Reprogramming
}

\author{
Julia Mester-Tonczar ${ }^{1}$, Ena Hašimbegović ${ }^{1}$, Andreas Spannbauer ${ }^{1}$, Denise Traxler', \\ Nina Kastner ${ }^{1}$, Katrin Zlabinger ${ }^{1}$, Patrick Einzinger ${ }^{2}$, Noemi Pavo', Georg Goliasch ${ }^{1}$ and \\ Mariann Gyöngyösi ${ }^{1 *}$ \\ ${ }^{1}$ Division of Cardiology, Department of Internal Medicine II, Medical University of Vienna, Vienna, Austria, \\ ${ }^{2}$ Research Unit of Information and Software Engineering, Institute of Information Systems Engineering, Vienna University of \\ Technology, Vienna, Austria
}

OPEN ACCESS

Edited by:

Reinier Boon,

Goethe University Frankfurt,

Germany

Reviewed by:

Meijing Wang,

Indiana University Bloomington

School of Medicine,

United States

Hamed Mirzaei,

Kashan University of Medical

Sciences, Iran

*Correspondence:

Mariann Gyöngyösi

mariann.gyongyosi@meduniwien.ac.at

Specialty section:

This article was submitted to Clinical and Translational Physiology,

a section of the journal

Frontiers in Physiology

Received: 06 July 2020 Accepted: 02 September 2020 Published: 29 September 2020

Citation:

Mester-Tonczar J, Hašimbegović E, Spannbauer A, Traxler D, Kastner N,

Zlabinger $K$, Einzinger $P$, Pavo N, Goliasch G and Gyöngyösi M (2020)

Circular RNAs in Cardiac

Regeneration: Cardiac Cell

Proliferation, Differentiation, Survival, and Reprogramming.

Front. Physiol. 11:580465.

doi: 10.3389/fphys.2020.580465
Circular RNAs (circRNAs) are classified as long non-coding RNAs (IncRNAs) that are characterized by a covalent closed-loop structure. This closed-loop shape is the result of a backsplicing event in which the 3 ' and 5 ' splice sites are ligated. Through the lack of 3' poly(A) tails and 5' cap structures, circRNAs are more stable than linear RNAs because these adjustments make the circular loop less susceptible to exonucleases. The majority of identified circRNAs possess cell- and tissue-specific expression patterns. In addition, high-throughput RNA-sequencing combined with novel bioinformatics algorithms revealed that circRNA sequences are often conserved across different species suggesting a positive evolutionary pressure. Implicated as regulators of protein turnover, micro RNA (miRNA) sponges, or broad effectors in cell differentiation, proliferation, and senescence, research of circRNA has increased in recent years. Particularly in cardiovascular research, circRNArelated discoveries have opened the door for the development of potential diagnostic and therapeutic tools. Increasing evidence links deviating circRNA expression patterns to various cardiovascular diseases including ischemic heart failure. In this mini-review, we summarize the current state of knowledge on circRNAs in cardiac regeneration with a focus on cardiac cell proliferation, differentiation, cardiomyocyte survival, and cardiac reprogramming.

Keywords: circular RNAs, cardiac regeneration, cardiac cell proliferation , cardiac reprogramming, ischemic heart failure, cardiovascular disease

\section{INTRODUCTION}

Approximately $98 \%$ of the human genome is comprised of non-coding RNA (ncRNA) transcripts (Bär et al., 2020). Nevertheless, protein-coding genes remain the most well-studied sequences in the mammalian genome (Esteller, 2011). However, it became apparent that ncRNAs are crucially involved in a wide array of physiological and pathophysiological processes (Brennecke et al., 2003; $\mathrm{Xu}$ et al., 2003; Abbaszadeh-Goudarzi et al., 2020; Hashemian et al., 2020; Yousefi et al., 2020). One recently re-discovered class of ncRNAs is circRNAs. 
The first description of circRNA coding organisms stems from 1976, when Sanger et al. examined viroids, circular singlestranded RNA pathogens of higher plants (Sanger et al., 1976). In eukaryotes, circRNAs were first detected in immortalized cervical cancer HeLa cells in 1979 using electron microscopy (Hsu and Coca-Prados, 1979). Until the 1990s, circRNAs were widely considered to be little more than splicing by-products or splicing errors (Capel et al., 1993; Cocquerelle et al., 1993). Since 2012, advances in high-throughput RNA-sequencing technology have identified circRNAs to be widespread, abundant and conserved across different species (Salzman et al., 2012, 2013; Jeck et al., 2013; AbouHaidar et al., 2014). Several circRNAs have shown a high degree of orthology between species as different as mice, pigs, and humans (Jeck et al., 2013).

CircRNAs were previously assumed to be present in low intracellular concentrations compared to conventional splicing products. However, recent analyses detected circRNAs in several human cell types and even indicated that they constitute a significant portion of the total spliced genetic product, varying between different cell types (Salzman et al., 2012). For some genes, the circular variant was also found to be more highly transcribed than the linear version (Salzman et al., 2012; Lasda and Parker, 2016).

In this mini-review, we provide a short overview on circRNA biogenesis and their mechanism of action in general. Further, we will summarize the newest findings regarding their role in cardiovascular diseases (CVD), in cardiomyocyte proliferation, differentiation, survival, and cardiac reprogramming. In conclusion, we outline current approaches to give insights into circRNAs in diagnostic and therapeutic settings, while considering state of the art methods, their limitations, and future challenges.

\section{TO COME FULL CIRCLE: BIOGENESIS OF CircRNAs}

Canonical splicing is the default mode of splicing for linear pre-mRNA transcripts. In canonical splicing, an upstream (5') splice donor site is joined with a downstream (3') splice acceptor site (Ng et al., 2004). CircRNAs, however, are formed through non-canoncial splicing, where a downstream donor site is ligated with an upstream splice acceptor site (Braun et al., 1996). This type of splicing leads to the formation of a backsplice junction in circRNAs which therefore lack a 5' to 3 ' directionality. The resulting products are a covalently closed circRNA and an additional linear transcript with skipped exons that is subject to fast degradation (Chen and Yang, 2015).

CircRNAs have longer half-lives than linear RNAs because their closed-loop structure is resistant to exonucleases, which typically require a $3^{\prime}$ or $5^{\prime}$ end to initiate degradation (Jeck et al., 2013; Lasda and Parker, 2014; Enuka et al., 2016). The stability and the low degradation rates may imply that accumulation, rather than high production rates are accountable for the measured circRNA levels. The first sets of data regarding the expression patterns of circRNAs are becoming available. Although there is a general tendency for them to be expressed on a level similar to their linear counterpart, there are several exceptions, including the highly expressed circRNAs correlated to Titin (TTN) and ryanodine receptor 2 (RYR2; Tan et al., 2017). Further differences between circRNAs and linear RNAs are shown in Table 1.

\section{MECHANISM OF ACTION}

The length of circRNAs ranges from a few hundred to thousands of nucleotides (Chen, 2016) and their functions are versatile. Most studies on circRNAs focus on their ability to act as miRNA sponges, whereby they inhibit miRNA-mRNA binding (Hansen et al., 2013; Memczak et al., 2013). Further, study by Li et al. (2015) revealed that exon-intron circRNAs (EIcircRNAs) regulate gene expression in the nucleus by increasing the expression of their parental genes via cis-mediated mechanisms. In addition, intron-containing circRNAs (ciRNAs) have been shown to act as regulators of RNA polymerase II in cells by associating with the machinery responsible for the Polymerase II elongation and positively regulating its transcription (Zhang et al., 2013; Holdt et al., 2018). Even though they are generally classified as ncRNAs, studies have identifies a subset of circRNAs, which can be translated in a cap-independent manner (Legnini et al., 2017; Pamudurti et al., 2017). However, the exact mechanism of circRNA translation is yet to be fully elucidated. The majority of identified circRNAs are stably expressed, with cell- and tissuespecific expression patterns (Salzman et al., 2013; Ji et al., 2019). Because of the ability of cells to distinguish between endogenous and exogenous circRNAs based on the intronic sequence that initiates the circularization of the RNA during splicing (Chen et al., 2017), it follows that the recognition and degradation of invasive circRNAs is a regulated response of the immune system, with implications for autoimmune diseases (Chen et al., 2017; Zhong et al., 2019). Further pathologic conditions, in which their regulatory mechanisms have been thoroughly investigated include cancer (Shabaninejad et al., 2019; Naeli et al., 2020), neurologic disorders (Memczak et al., 2013), diabetes (AbbaszadehGoudarzi et al., 2020), and CVD (Wang et al., 2016; Werfel et al., 2016).

TABLE 1 | Main differences between circular RNAs (circRNAs) and linear RNAs.

\begin{tabular}{|c|c|c|c|}
\hline & CircRNA & Linear RNA & References \\
\hline $\begin{array}{l}\text { Deep sequencing } \\
\text { required }\end{array}$ & Yes & Yes & $\begin{array}{l}\text { Salzman et al., 2012; Cooper } \\
\text { et al., 2018; Das et al., } 2019\end{array}$ \\
\hline Stability & Yes & No & $\begin{array}{l}\text { Jeck et al., 2013; Lasda and } \\
\text { Parker, 2014; Enuka et al., } 2016\end{array}$ \\
\hline $\begin{array}{l}\text { Exonuclease } \\
\text { resistant* }\end{array}$ & Yes & No & $\begin{array}{l}\text { Suzuki et al., 2006; Vincent and } \\
\text { Deutscher, 2006; Salzman et al., } \\
2012\end{array}$ \\
\hline Backsplice junction & Yes & No & Das et al., 2019 \\
\hline 5' cap structure & No & Yes & Holdt et al., 2018 \\
\hline 3' poly(A)-tails & No & Yes & Holdt et al., 2018 \\
\hline $\begin{array}{l}\text { Length in } \\
\text { basepairs (bp) }\end{array}$ & $>200$ & 21 to $>200$ & Ding et al., 2018 \\
\hline
\end{tabular}

*Some circRNAs are not exonuclease resistant (Szabo and Salzman, 2016; Legnini et al., 2017). 


\section{CircRNAs IN CVD}

In recent years, the link between circRNAs and CVD has been studied intensively and indicates their involvement in the CVD pathogenesis (Viereck and Thum, 2017; Gurha, 2019; Huang et al., 2020). Since many circRNAs are highly conserved across species, animal models can be used to infer the role of circRNAs in human CVD (Werfel et al., 2016; Tan et al., 2017).

Two of the most well-known circRNAs with key functions in the heart, namely heart-related circRNA (HRCR) and CDR1as act as miRNA sponges (Huang et al., 2020). The conveyed effect depends on the "sponged" miRNA: while HRCR has an attenuating effect on hypertrophy by sponging miR-223 (Wang et al., 2016), CDRlas seems to amplify post-myocardial infarction (MI) ischemic damage in mice through sponging miR-7 (Geng et al., 2016). Interestingly, in a porcine model of MI increased expression of CDRlas was associated with reduced infarct size and increased left ventricular (LV) and right ventricular (RV) function (Mester-Tonczar et al., 2020). This might indicate interspecies differences in the role of CDRlas in CVD.

CircRNAs have been detected in peripheral fluids such as whole blood and plasma, where their increased chemical stability may be an advantage for their use as clinical biomarkers of diseases (Gurha, 2019). A study from 2019 established a circRNA-miRNA-mRNA network and identified circYOD-1 as a circulating biomarker for coronary artery disease (Miao et al., 2019). A study by Wu et al. (2019) identified three differentialy regulated circRNAs in pediatric patients with congenital heart disease: hsa_circRNA_004183, hsa_circRNA_ 079265 and hsa_circRNA_105039. Another study reported several circRNAs linked to hypertrophic cardiomyopathy (HCM) in humans. Three of the investigated circRNAs, DNAJC6, TMEM56, and MBOAT2 were found in serum and can help distinguish healthy from HCM patients. Furthermore, DNAJC6 and TMEM56 could serve as indicators for disease severity in patients with obstructive HCM (Sonnenschein et al., 2019). Identifying differentially regulated circRNAs through newly available sequencing technologies is the first step in planning further research with circRNAs.

\section{CircRNAS IN CARDIAC REGENERATION: CARDIOMYOCYTE PROLIFERATION, DIFFERENTIATION, AND SURVIVAL}

Despite essential advances in cardiac regenerative medicine, regeneration following myocardial ischemia still imposes many obstacles. Until recently, stem cell treatments, gene therapy, cell-based gene therapy, or the use of paracrine factors as regenerative cocktails of sorts represented the main focus of regenerative cardiology, yet the efficacy of such treatments failed to live up to expectations (Gyöngyösi et al., 2018). Regardless of the underlying etiology and the multitude of approaches for revascularizing or repairing ischemic tissue, reducing further damage outside the infarcted zone, and protecting the remaining healthy myocardium, the lack of clinically meaningful regenerative potential of mature cardiomyocytes and their replacement with non-functional scar results in an irreversible loss of functional tissue. Thus, new research directions focused on circRNAs in cardiac cell proliferation, differentiation, and cardiac survival should be examined.

The following part of the review focuses on current studies involving circRNAs within the context of the growing field of research on cardiac reprogramming. The natural starting point for any such investigations is the exploration of circRNA regulation in the growing and developing heart. Further investigations have focused on the differential regulation of circRNA transcripts in mesenchymal stem cells (MSCs) and induced pluripotent stem cells (iPSCs) during their experimentally induced differentiation into cardiomyocytes. Lastly, we discuss the literature on circRNA expression following MI.

\section{CircRNA Expression Patterns During Cardiac Development}

In 2017, Li et al. examined the stage specific expression of lncRNAs and circRNAs in the undifferentiated mesoderm, cardiac progenitor, and definitive cardiomyocyte stages of cardiomyocyte development. The analysis uncovered important circRNA regulators, including circ-TTN, and a co-expression pattern of the circRNAs with genes such as MYL4, whose mutation has been linked to cardiac structural and electrical abnormalities (Li et al., 2017).

A 2017 study by $\mathrm{Xu}$ et al. measured the circRNA expression patterns in six human tissues, including the heart, and compared them to the expression patterns of the corresponding fetal tissue. As a general rule, they found circRNAs to be more highly expressed in the fetal stage of development. CircSLC8A1 was found to have a heart-specific upregulation pattern and might be a viable target for further research (Xu et al., 2017).

\section{CircRNAs in MSCs}

MSCs not only have the ability to differentiate into cardiomyocytes, but can also recruit resident cardiac stem cells and secrete a variety of factors that could be useful in terms of cardiac regeneration (Williams and Hare, 2011).

A total of 226 differentially regulated circRNAs were discovered regarding differentiation of umbilical cord-derived human MSCs (huMSCs) into cardiomyocytes, with the most highly differentially regulated circRNAs related to differentiation and proliferation pathways, including the Wnt pathway (Ruan et al., 2019).

Another circRNA involved in the transcriptional preservation of stem cell identity is circFOXP1, whose silencing reduces huMSC growth and proliferation. MiR-17-3p and miR-127-5p are among the miRNAs sponged by circFOXP1. MiR-17-3p and $\mathrm{miR}-127-3 \mathrm{p}$ have a role in epidermal growth factor receptor (EGFR) and canonical Wnt signaling, an axis that allows them to interact in growth and survival pathways, as well as influencing the amount of differentiated MSCs (Cherubini et al., 2019).

CircRNA CDRlas has also been found to be abundant in huMSCs. The increase of huMSC proliferation, secretion, and differentiation induced by $3,3^{\prime}$-diindolylmethane also increased 
the abundance of CDR1as, whereas the knockdown of CDR1as reduces their proliferation and differentiation capacity, which are essential for their regenerative potential (Yang et al., 2019).

\section{CircRNAs in iPSCs}

Early attempts at inducing cardiac regeneration focused on the stimulation of stem cell and cardiac progenitor cell populations naturally residing in the heart (Cai and Molkentin, 2017; Tzahor and Poss, 2017). However, this approach is limited by the small number of these c-kit+ and Sca-1+ cells in the adult myocardium and their replicative and functional limitations in replacing cardiomyocytes and achieving significant physiological effects (van Berlo and Molkentin, 2014; Cai and Molkentin, 2017).

Other approaches focused on the application of autologous or allogeneic somatic or embryonic stem cells. However, immunogenicity, engraftment, differentiation, tumogenicity, and ethical concerns were common issues with these methods.

A new era in the field of regenerative research, particularly in cardiac regeneration, was ushered in by Takahashi (Takahashi and Yamanaka, 2006; Takahashi et al., 2007). His first study revealed that differentiated somatic mouse cells can be reprogramed through the use of transcription factors to revert back to a pluripotent state (Takahashi and Yamanaka, 2006). The second study managed to replicate the same results in humans, inducing pluripotency in human dermal fibroblasts (Takahashi et al., 2007). These iPSCs became the focus of a multitude of studies and remain a very active field of research to this day. In a human induced pluripotent stem cell derived cardiomyocyte model, researchers investigated the differential regulation of circRNAs in cardiac development and identified 384 circRNAs that are specific to the setting of developing cardiomyocytes. Furthermore, multiple circRNAs were identified, which are relatively abundant or depleted in relation to the respective linear transcript over the course of cardiomyocyte differentiation, exemplified by circCDYL and circ-SMARCA5 (Siede et al., 2017).

A breakthrough study by Ieda et al. (2010) demonstrated that transformation of fibroblasts into functioning cardiomyocytes was successful, leading to the concept that differentiated somatic cells can directly be programed into another cell type without transitioning through the pluripotent stage.

\section{CircRNAs Following MI: A Story of Angiogenesis, Pluripotency, Proliferation, and Altered Outcomes}

It is often difficult to speculate whether a single circRNA can convey a meaningful regeneration following a $\mathrm{MI}$, since many circRNAs act on multiple pathways.

In 2019, Huang et al. identified super-enhancer associated circRNAs that could be relevant in regenerative pathways and further examined the role of circRNA Nfix in the regenerative capacity of the myocardium following a MI. On the one hand, circNfix knockdown not only induced increased cardiomyocyte proliferation in a murine model, but also resulted in improved angiogenesis, reduced infarcted and fibrotic area and improved cardiac functional parameters following an infarction. An induced overexpression of circNfix on the other hand decreased the ability of cardiomyocytes to proliferate in vivo. All of these potentially highly therapeutically relevant effects were found to be conveyed through promoting $\mathrm{Ybx}$ degradation and sponging miR-214 (Huang et al., 2019).

CircCDYL was the focus of a 2020 study on cardiac regeneration conducted by Zhang et al. This research group investigated circCDYL in adult mouse cardiomyocytes and in murine myocardium, and showed that overexpression of circCDYL in vitro promotes cardiomyocyte proliferation, whereas downregulation of circCDYL inhibits their proliferation. In vivo overexpression of circCDYL led to increase in ejection fraction (EF) in mice, whereas downregulation of circCDYL lowered the EF (Zhang et al., 2020b).

CircRNAs are also involved in post-infarction fibrotic remodeling that results in heart failure following $\mathrm{MI}$ in the long run. Zhu et al. (2019) investigated the role of circNFIB in cardiac fibrosis in a mouse model of MI and reported that circNFIB inhibition promotes adult fibroblast proliferation. Additionally, the sponge activity of circNFIB on miR-433 was also identified.

CircRNAs are often the topic of multidisciplinary research. CircHIPK3 is an example of a circRNA involved in cellular growth that has simultaneously been implicated in the growth, proliferation, and metastasis of cancer (Zheng et al., 2016; Zeng et al., 2018). CircHIPK3 is also relatively abundant in the fetal and neonatal myocardium of mice (Si et al., 2020, p. 3). This motivated an investigation into its potential regeneration-inducing capacity in the myocardium in vitro and in vivo. CircHIPK3 is perhaps the perfect example to illustrate the sheer multitude of effects that can be exhibited by a single circRNA. Si et al. (2020) demonstrated that the overexpression adenoviral vectorassociated circHIPK3 induces coronary artery endothelial proliferation, increases cardiomyocyte proliferation, promotes angiogenesis, and decreases fibrosis in the zone surrounding the infarction area in adult mice. Whereas the pro-angiogenic properties seem to be conveyed through a miRNA-133a sponging effect, the effects on the cardiomyocytes were found to be achieved through an increase in the stability of the Notch1 intracellular domain. Table 2 summarizes circRNAs involved in cardiomyocyte proliferation, differentiation, and cardiac reprogramming.

\section{AREAS OF APPLICATION}

As CircRNAs are involved in cell differentiation and proliferation as well as regulating transcription (Bose and Ain, 2018) and translation (Pamudurti et al., 2017), their potential applications are plentiful. CircRNAs are currently the focus of a number of biomarker studies (Ouyang et al., 2017; Zhao et al., 2017; Li et al., 2018), due to their chemical stability compared to other RNA molecules (Jeck et al., 2013). Wesselhoeft et al. (2018) demonstrated the use of circRNAs for robust and stable translation in eukaryotic cells. This innovation offers great possibilities for obtaining stable protein production for biotechnological use. Their development can be used as an alternative to mRNA translation as mRNAs display relatively short half-lives compared to circRNAs. Another area of application is the construction of 
TABLE 2 | CircRNAs and their role in cardiac cell proliferation, differentiation, and cardiac reprogramming.

\begin{tabular}{|c|c|c|c|c|c|}
\hline CircRNA & Regulation & Mechanism & Consequence & $\begin{array}{l}\text { Type of sample (number of } \\
\text { samples) }\end{array}$ & References \\
\hline HRCR & Overexpression & miR-223 sponge & $\begin{array}{l}\text { Repression of cardiac hypertrophy and } \\
\text { HF in mice }\end{array}$ & Mice hearts ( $n=4-8$ /group) & Wang et al., 2016 \\
\hline CDR1as & $\begin{array}{l}\text { Increased } \\
\text { expression }\end{array}$ & Currently unknown & $\begin{array}{l}\text { Reduced infarct size and positive } \\
\text { influence on LV and RV function }\end{array}$ & Pig hearts ( $n=5$ /group) & $\begin{array}{l}\text { Mester-Tonczar et al., } \\
2020\end{array}$ \\
\hline circFOXP1 & $\begin{array}{l}\text { Silencing } \\
\text { circFOXP1 }\end{array}$ & $\begin{array}{l}\text { miR-17-3p sponge and miR- } \\
127-5 p \text { sponge resulting in the } \\
\text { modulation of non-canonical } \\
\text { Wnt and EGFR pathways }\end{array}$ & $\begin{array}{l}\text { Reduction of huMSC growth and } \\
\text { proliferation }\end{array}$ & huMSCs & Cherubini et al., 2019 \\
\hline circCDYL & Overexpression & Currently unknown & Improved heart function after AMI & Hypoxic cardiomyocytes & Zhang et al., 2020b \\
\hline \multirow[t]{2}{*}{ circNFIB } & \multirow[t]{2}{*}{ Overexpression } & \multirow[t]{2}{*}{ miR-433 sponge } & \multirow{2}{*}{$\begin{array}{l}\text { Attenuation of cardiac fibroblast } \\
\text { proliferation }\end{array}$} & Mice hearts ( $n=5-6 /$ group) & \multirow[t]{2}{*}{ Zhu et al., 2019} \\
\hline & & & & $\begin{array}{l}\text { TGF-beta treated cardiac mice } \\
\text { fibroblasts }\end{array}$ & \\
\hline circHIPK3 & Overexpression & $\begin{array}{l}\text { Increased N1ICD acetylation } \\
\text { and miR-133a sponge }\end{array}$ & $\begin{array}{l}\text { Induction of coronary artery endothelial } \\
\text { cell proliferation, promoted } \\
\text { cardiomyocyte proliferation and } \\
\text { angiogenesis, and decreased fibrosis } \\
\text { after Ml }\end{array}$ & Mouse model of MI $(n=6)$ & Si et al., 2020 \\
\hline
\end{tabular}

huMSCs, human mesenchymal stem cells; HF, heart failure; LV function, left ventricular function; MI, myocardial infarction; N1ICD, Notch1 intracellular domain; RV function, right ventricular function. The number of samples varies in these studies, because the research groups used varying numbers of animals for each of their experiments.

synthetic miRNA sponges. Liu et al. (2018) demonstrated a successful designing of a synthetic sponge constructed out of a circularized product targeting miR-21. As a consequence, gastric carcinoma cell proliferation was inhibited. In the past 3 years, other studies also reported the engineering of cost-effective artificial miRNA sponges (Rossbach, 2019; Wang et al., 2019). These examples illustrate the potential of circRNA for developing biomarkers, tools for circRNAs translation, and therapeutic potential for regulating cell proliferation.

\section{ChALLENGES IN CircRNA RESEARCH}

Since most circRNAs show low expression patterns (Zhang et al., 2020a), their detectability requires state of the art methods. For the detection of novel circRNAs deep-sequencing paired with bioinformatics algorithms specialized on detecting backsplice junctions is required. Several algorithms exist, each with its own advantages and disadvantages. Some algorithms like KNIFE, CIRCexplorer, and CIRI achieved the best sensitivity, whereas others like UROBORUS can detect low expression levels of circRNAs in rRNA depleted samples without RNase $\mathrm{R}$ digestion (Chen et al., 2020). No poly(A) enrichment should be performed before RNA-sequencing and a read length of at least 100 basepairs is recommended to accurately align the reads with the backsplice junction (Kristensen et al., 2019). Some laboratories propose performing an RNase R digestion for circRNAs, however, not all circRNAs are resistant to RNase R (Szabo and Salzman, 2016; Legnini et al., 2017). Instead of using RNase R in circRNA research, one could use divergent primers spanning the backsplice junction and confirm the circular transcript with Sanger sequencing. Similarly, RNA-sequencing data can be validated through Sanger sequencing as well.

\section{CONCLUSION}

In this review, we summarized some of the recent findings on the topic of circRNAs and their role in cardiomyocyte proliferation, differentiation, survival, and cardiovascular regeneration. Given the fact that circRNAs were only recently re-discovered compared to other ncRNAs, the full potential of circRNAs has yet to be elaborated.

\section{AUTHOR CONTRIBUTIONS}

JM-T wrote the manuscript and put the tables together. MG, $\mathrm{EH}$, and AS helped in literature search and assisted with writing the manuscript. DT, NK, KZ, PE, NP, and GG edited the manuscript and the tables and helped to structure and revise the manuscript. All authors contributed to the article and approved the submitted version. 


\section{REFERENCES}

Abbaszadeh-Goudarzi, K., Radbakhsh, S., Pourhanifeh, M. H., Khanbabaei, H., Davoodvandi, A., Fathizadeh, H., et al. (2020). Circular RNA and diabetes: epigenetic regulator with diagnostic role. Curr. Mol. Med. 20, 516-526. doi: $10.2174 / 1566524020666200129142106$

AbouHaidar, M. G., Venkataraman, S., Golshani, A., Liu, B., and Ahmad, T. (2014). Novel coding, translation, and gene expression of a replicating covalently closed circular RNA of 220 nt. Proc. Natl. Acad. Sci. U. S. A. 111, 14542-14547. doi: 10.1073/pnas.1402814111

Bär, C., Chatterjee, S., Falcão Pires, I., Rodrigues, P., Sluijter, J. P. G., Boon, R. A., et al. (2020). Non-coding RNAs: update on mechanisms and therapeutic targets from the ESC working groups of myocardial function and cellular biology of the heart. Cardiovasc. Res. 116, 1805-1819. doi: 10.1093/cvr/cvaa195

Bose, R., and Ain, R. (2018). Regulation of transcription by circular RNAs. Adv. Exp. Med. Biol. 1087, 81-94. doi: 10.1007/978-981-13-1426-1_7

Braun, S., Domdey, H., and Wiebauer, K. (1996). Inverse splicing of a discontinuous pre-mRNA intron generates a circular exon in a HeLa cell nuclear extract. Nucleic Acids Res. 24, 4152-4157. doi: 10.1093/nar/24.21.4152

Brennecke, J., Hipfner, D. R., Stark, A., Russell, R. B., and Cohen, S. M. (2003). Bantam encodes a developmentally regulated microRNA that controls cell proliferation and regulates the proapoptotic gene hid in Drosophila. Cell 113, 25-36. doi: 10.1016/s0092-8674(03)00231-9

Cai, C. -L., and Molkentin, J. D. (2017). The elusive progenitor cell in cardiac regeneration: Slip Slidin' Away. Circ. Res. 120, 400-406. doi: 10.1161/ CIRCRESAHA.116.309710

Capel, B., Swain, A., Nicolis, S., Hacker, A., Walter, M., Koopman, P., et al. (1993). Circular transcripts of the testis-determining gene Sry in adult mouse testis. Cell 73, 1019-1030. doi: 10.1016/0092-8674(93)90279-y

Chen, L. -L. (2016). The biogenesis and emerging roles of circular RNAs. Nat. Rev. Mol. Cell Biol. 17, 205-211. doi: 10.1038/nrm.2015.32

Chen, Y. G., Kim, M. V., Chen, X., Batista, P. J., Aoyama, S., Wilusz, J. E., et al. (2017). Sensing self and foreign circular RNAs by intron identity. Mol. Cell 67, 228.e5-238.e5. doi: 10.1016/j.molcel.2017.05.022

Chen, L., Wang, C., Sun, H., Wang, J., Liang, Y., Wang, Y., et al. (2020). The bioinformatics toolbox for circRNA discovery and analysis. Brief. Bioinform. doi: 10.1093/bib/bbaa001 [Epub ahead of print]

Chen, L. -L., and Yang, L. (2015). Regulation of circRNA biogenesis. RNA Biol. 12, 381-388. doi: 10.1080/15476286.2015.1020271

Cherubini, A., Barilani, M., Rossi, R. L., Jalal, M. M. K., Rusconi, F., Buono, G., et al. (2019). FOXP1 circular RNA sustains mesenchymal stem cell identity via microRNA inhibition. Nucleic Acids Res. 47, 5325-5340. doi: 10.1093/ nar/gkz199

Cocquerelle, C., Mascrez, B., Hétuin, D., and Bailleul, B. (1993). Mis-splicing yields circular RNA molecules. FASEB J. 7, 155-160. doi: 10.1096/ fasebj.7.1.7678559

Cooper, D. A., Cortés-López, M., and Miura, P. (2018). Genome-wide circRNA profiling from RNA-seq data. Methods Mol. Biol. 1724, 27-41. doi: 10.1007/978-1-4939-7562-4_3

Das, A., Rout, P. K., Gorospe, M., and Panda, A. C. (2019). Rolling circle cDNA synthesis uncovers circular RNA splice variants. Int. J. Mol. Sci. 20:3988. doi: 10.3390/ijms20163988

Ding, X., Zhang, S., Li, X., Feng, C., Huang, Q., Wang, S., et al. (2018). Profiling expression of coding genes, long noncoding RNA, and circular RNA in lung adenocarcinoma by ribosomal RNA-depleted RNA sequencing. FEBS Open Bio 8, 544-555. doi: 10.1002/2211-5463.12397

Enuka, Y., Lauriola, M., Feldman, M. E., Sas-Chen, A., Ulitsky, I., and Yarden, Y. (2016). Circular RNAs are long-lived and display only minimal early alterations in response to a growth factor. Nucleic Acids Res. 44, 1370-1383. doi: 10.1093/ nar/gkv1367

Esteller, M. (2011). Non-coding RNAs in human disease. Nat. Rev. Genet. 12, 861-874. doi: $10.1038 / \operatorname{nrg} 3074$

Geng, H. -H., Li, R., Su, Y. -M., Xiao, J., Pan, M., Cai, X. -X., et al. (2016). The circular RNA Cdrlas promotes myocardial infarction by mediating the regulation of miR-7a on its target genes expression. PLoS One 11:e0151753. doi: 10.1371/journal.pone.0151753

Gurha, P. (2019). Noncoding RNAs in cardiovascular diseases. Curr. Opin. Cardiol. 34, 241-245. doi: 10.1097/HCO.0000000000000615
Gyöngyösi, M., Haller, P. M., Blake, D. J., and Martin Rendon, E. (2018). Meta-analysis of cell therapy studies in heart failure and acute myocardial infarction. Circ. Res. 123, 301-308. doi: 10.1161/CIRCRESAHA.117. 311302

Hansen, T. B., Jensen, T. I., Clausen, B. H., Bramsen, J. B., Finsen, B., Damgaard, C. K., et al. (2013). Natural RNA circles function as efficient microRNA sponges. Nature 495, 384-388. doi: 10.1038/nature11993

Hashemian, S. M., Pourhanifeh, M. H., Fadaei, S., Velayati, A. A., Mirzaei, H., and Hamblin, M. R. (2020). Non-coding RNAs and exosomes: their role in the pathogenesis of sepsis. Mol. Ther. Nucleic Acids 21, 51-74. doi: 10.1016/j. omtn.2020.05.012

Holdt, L. M., Kohlmaier, A., and Teupser, D. (2018). Molecular roles and function of circular RNAs in eukaryotic cells. Cell. Mol. Life Sci. 75, 1071-1098. doi: $10.1007 / \mathrm{s} 00018-017-2688-5$

Hsu, M. T., and Coca-Prados, M. (1979). Electron microscopic evidence for the circular form of RNA in the cytoplasm of eukaryotic cells. Nature 280, 339-340. doi: 10.1038/280339a0

Huang, C. -K., Kafert-Kasting, S., and Thum, T. (2020). Preclinical and clinical development of noncoding RNA therapeutics for cardiovascular disease. Circ. Res. 126, 663-678. doi: 10.1161/CIRCRESAHA.119.315856

Huang, S., Li, X., Zheng, H., Si, X., Li, B., Wei, G., et al. (2019). Loss of super-enhancer-regulated circRNA Nfix induces cardiac regeneration after myocardial infarction in adult mice. Circulation 139, 2857-2876. doi: 10.1161/ CIRCULATIONAHA.118.038361

Ieda, M., Fu, J. -D., Delgado-Olguin, P., Vedantham, V., Hayashi, Y., Bruneau, B. G., et al. (2010). Direct reprogramming of fibroblasts into functional cardiomyocytes by defined factors. Cell 142, 375-386. doi: 10.1016/j.cell.2010.07.002

Jeck, W. R., Sorrentino, J. A., Wang, K., Slevin, M. K., Burd, C. E., Liu, J., et al. (2013). Circular RNAs are abundant, conserved, and associated with ALU repeats. RNA 19, 141-157. doi: 10.1261/rna.035667.112

Ji, P., Wu, W., Chen, S., Zheng, Y., Zhou, L., Zhang, J., et al. (2019). Expanded expression landscape and prioritization of circular RNAs in mammals. Cell Rep. 26, 3444.e5-3460.e5. doi: 10.1016/j.celrep.2019.02.078

Kristensen, L. S., Andersen, M. S., Stagsted, L. V. W., Ebbesen, K. K., Hansen, T. B., and Kjems, J. (2019). The biogenesis, biology and characterization of circular RNAs. Nat. Rev. Genet. 20, 675-691. doi: 10.1038/s41576-019-0158-7

Lasda, E., and Parker, R. (2014). Circular RNAs: diversity of form and function. RNA 20, 1829-1842. doi: 10.1261/rna.047126.114

Lasda, E., and Parker, R. (2016). Circular RNAs co-precipitate with extracellular vesicles: a possible mechanism for circRNA clearance. PLoS One 11:e0148407. doi: 10.1371/journal.pone.0148407

Legnini, I., Di Timoteo, G., Rossi, F., Morlando, M., Briganti, F., Sthandier, O., et al. (2017). Circ-ZNF609 is a circular RNA that can be translated and functions in myogenesis. Mol. Cell 66, 22.e9-37.e9. doi: 10.1016/j.molcel.2017. 02.017

Li, Z., Huang, C., Bao, C., Chen, L., Lin, M., Wang, X., et al. (2015). Exonintron circular RNAs regulate transcription in the nucleus. Nat. Struct. Mol. Biol. 22, 256-264. doi: 10.1038/nsmb.2959

Li, H., Li, K., Lai, W., Li, X., Wang, H., Yang, J., et al. (2018). Comprehensive circular RNA profiles in plasma reveals that circular RNAs can be used as novel biomarkers for systemic lupus erythematosus. Clin. Chim. Acta 480, 17-25. doi: 10.1016/j.cca.2018.01.026

Li, Y., Zhang, J., Huo, C., Ding, N., Li, J., Xiao, J., et al. (2017). Dynamic organization of IncRNA and circular RNA regulators collectively controlled cardiac differentiation in humans. EBioMedicine 24, 137-146. doi: 10.1016/j. ebiom.2017.09.015

Liu, X., Abraham, J. M., Cheng, Y., Wang, Z., Wang, Z., Zhang, G., et al. (2018). Synthetic circular RNA functions as a miR-21 sponge to suppress gastric carcinoma cell proliferation. Mol. Ther. Nucleic Acids 13, 312-321. doi: 10.1016/j.omtn.2018.09.010

Memczak, S., Jens, M., Elefsinioti, A., Torti, F., Krueger, J., Rybak, A., et al. (2013). Circular RNAs are a large class of animal RNAs with regulatory potency. Nature 495, 333-338. doi: 10.1038/nature11928

Mester-Tonczar, J., Winkler, J., Einzinger, P., Hasimbegovic, E., Kastner, N., Lukovic, D., et al. (2020). Association between circular RNA CDRlas and post-infarction cardiac function in pig ischemic heart failure: influence of the anti-fibrotic natural compounds bufalin and lycorine. Biomol. Ther 10:E1180. doi: 10.3390/biom10081180 
Miao, L., Yin, R. -X., Zhang, Q. -H., Liao, P. -J., Wang, Y., Nie, R. -J., et al. (2019). A novel circRNA-miRNA-mRNA network identifies circ-YOD1 as a biomarker for coronary artery disease. Sci. Rep. 9:18314. doi: 10.1038/ s41598-019-54603-2

Naeli, P., Pourhanifeh, M. H., Karimzadeh, M. R., Shabaninejad, Z., Movahedpour, A., Tarrahimofrad, H., et al. (2020). Circular RNAs and gastrointestinal cancers: epigenetic regulators with a prognostic and therapeutic role. Crit. Rev. Oncol. Hematol. 145:102854. doi: 10.1016/j.critrevonc. 2019.102854

Ng, B., Yang, F., Huston, D. P., Yan, Y., Yang, Y., Xiong, Z., et al. (2004). Increased noncanonical splicing of autoantigen transcripts provides the structural basis for expression of untolerized epitopes. J. Allergy Clin. Immunol. 114, 1463-1470. doi: 10.1016/j.jaci.2004.09.006

Ouyang, Q., Wu, J., Jiang, Z., Zhao, J., Wang, R., Lou, A., et al. (2017). Microarray expression profile of circular RNAs in peripheral blood mononuclear cells from rheumatoid arthritis patients. Cell. Physiol. Biochem. 42, 651-659. doi: 10.1159/000477883

Pamudurti, N. R., Bartok, O., Jens, M., Ashwal-Fluss, R., Stottmeister, C., Ruhe, L., et al. (2017). Translation of CircRNAs. Mol. Cell 66, 9.e7-21.e7. doi: 10.1016/j.molcel.2017.02.021

Rossbach, O. (2019). Artificial circular RNA sponges targeting microRNAs as a novel tool in molecular biology. Mol. Ther. Nucleic Acids 17, 452-454. doi: 10.1016/j.omtn.2019.06.021

Ruan, Z. -B., Chen, G. -C., Zhang, R., and Zhu, L. (2019). Circular RNA expression profiles during the differentiation of human umbilical cord-derived mesenchymal stem cells into cardiomyocyte-like cells. J. Cell. Physiol. 234, 16412-16423. doi: 10.1002/jcp. 28310

Salzman, J., Chen, R. E., Olsen, M. N., Wang, P. L., and Brown, P. O. (2013). Cell-type specific features of circular RNA expression. PLoS Genet. 9:e1003777. doi: 10.1371/journal.pgen.1003777

Salzman, J., Gawad, C., Wang, P. L., Lacayo, N., and Brown, P. O. (2012). Circular RNAs are the predominant transcript isoform from hundreds of human genes in diverse cell types. PLoS One 7:e30733. doi: 10.1371/journal.pone.0030733

Sanger, H. L., Klotz, G., Riesner, D., Gross, H. J., and Kleinschmidt, A. K. (1976). Viroids are single-stranded covalently closed circular RNA molecules existing as highly base-paired rod-like structures. Proc. Natl. Acad. Sci. U. S. A. 73, 3852-3856. doi: 10.1073/pnas.73.11.3852

Shabaninejad, Z., Vafadar, A., Movahedpour, A., Ghasemi, Y., Namdar, A., Fathizadeh, H., et al. (2019). Circular RNAs in cancer: new insights into functions and implications in ovarian cancer. J. Ovarian Res. 12:84. doi: 10.1186/s13048-019-0558-5

Si, X., Zheng, H., Wei, G., Li, M., Li, W., Wang, H., et al. (2020). circRNA Hipk3 induces cardiac regeneration after myocardial infarction in mice by binding to Notch1 and miR-133a. Mol. Ther. Nucleic Acids 21, 636-655. doi: 10.1016/j.omtn.2020.06.024

Siede, D., Rapti, K., Gorska, A. A., Katus, H. A., Altmüller, J., Boeckel, J. N., et al. (2017). Identification of circular RNAs with host gene-independent expression in human model systems for cardiac differentiation and disease. J. Mol. Cell. Cardiol. 109, 48-56. doi: 10.1016/j.yjmcc.2017.06.015

Sonnenschein, K., Wilczek, A. L., de Gonzalo-Calvo, D., Pfanne, A., Derda, A. A., Zwadlo, C., et al. (2019). Serum circular RNAs act as blood-based biomarkers for hypertrophic obstructive cardiomyopathy. Sci. Rep. 9:20350. doi: 10.1038/ s41598-019-56617-2

Suzuki, H., Zuo, Y., Wang, J., Zhang, M. Q., Malhotra, A., and Mayeda, A. (2006). Characterization of RNase R-digested cellular RNA source that consists of lariat and circular RNAs from pre-mRNA splicing. Nucleic Acids Res. 34:e63. doi: 10.1093/nar/gkl151

Szabo, L., and Salzman, J. (2016). Detecting circular RNAs: bioinformatic and experimental challenges. Nat. Rev. Genet. 17, 679-692. doi: 10.1038/nrg.2016.114

Takahashi, K., Tanabe, K., Ohnuki, M., Narita, M., Ichisaka, T., Tomoda, K., et al. (2007). Induction of pluripotent stem cells from adult human fibroblasts by defined factors. Cell 131, 861-872. doi: 10.1016/j.cell.2007.11.019

Takahashi, K., and Yamanaka, S. (2006). Induction of pluripotent stem cells from mouse embryonic and adult fibroblast cultures by defined factors. Cell 126, 663-676. doi: 10.1016/j.cell.2006.07.024

Tan, W. L. W., Lim, B. T. S., Anene-Nzelu, C. G. O., Ackers-Johnson, M., Dashi, A., See, K., et al. (2017). A landscape of circular RNA expression in the human heart. Cardiovasc. Res. 113, 298-309. doi: 10.1093/cvr/ crw250
Tzahor, E., and Poss, K. D. (2017). Cardiac regeneration strategies: staying young at heart. Science 356, 1035-1039. doi: 10.1126/science.aam5894

van Berlo, J. H., and Molkentin, J. D. (2014). An emerging consensus on cardiac regeneration. Nat. Med. 20, 1386-1393. doi: 10.1038/nm.3764

Viereck, J., and Thum, T. (2017). Long noncoding RNAs in pathological cardiac remodeling. Circ. Res. 120, 262-264. doi: 10.1161/CIRCRESAHA.116. 310174

Vincent, H. A., and Deutscher, M. P. (2006). Substrate recognition and catalysis by the exoribonuclease RNase R. J. Biol. Chem. 281, 29769-29775. doi: 10.1074/jbc.M606744200

Wang, K., Long, B., Liu, F., Wang, J. -X., Liu, C. -Y., Zhao, B., et al. (2016). A circular RNA protects the heart from pathological hypertrophy and heart failure by targeting miR-223. Eur. Heart J. 37, 2602-2611. doi: 10.1093/ eurheartj/ehv713

Wang, Z., Ma, K., Cheng, Y., Abraham, J. M., Liu, X., Ke, X., et al. (2019). Synthetic circular multi-miR sponge simultaneously inhibits miR-21 and miR-93 in esophageal carcinoma. Lab. Investig. 99, 1442-1453. doi: 10.1038/ s41374-019-0273-2

Werfel, S., Nothjunge, S., Schwarzmayr, T., Strom, T. -M., Meitinger, T., and Engelhardt, S. (2016). Characterization of circular RNAs in human, mouse and rat hearts. J. Mol. Cell. Cardiol. 98, 103-107. doi: 10.1016/j.yjmcc.2016. 07.007

Wesselhoeft, R. A., Kowalski, P. S., and Anderson, D. G. (2018). Engineering circular RNA for potent and stable translation in eukaryotic cells. Nat. Commun. 9:2629. doi: 10.1038/s41467-018-05096-6

Williams, A. R., and Hare, J. M. (2011). Mesenchymal stem cells: biology, pathophysiology, translational findings, and therapeutic implications for cardiac disease. Circ. Res. 109, 923-940. doi: 10.1161/CIRCRESAHA.111.243147

Wu, J., Li, J., Liu, H., Yin, J., Zhang, M., Yu, Z., et al. (2019). Circulating plasma circular RNAs as novel diagnostic biomarkers for congenital heart disease in children. J. Clin. Lab. Anal. 33:e22998. doi: 10.1002/jcla.22998

Xu, P., Vernooy, S. Y., Guo, M., and Hay, B. A. (2003). The Drosophila microRNA Mir-14 suppresses cell death and is required for normal fat metabolism. Curr. Biol. 13, 790-795. doi: 10.1016/s0960-9822(03)00250-1

Xu, T., Wu, J., Han, P., Zhao, Z., and Song, X. (2017). Circular RNA expression profiles and features in human tissues: a study using RNA-seq data. BMC Genomics 18:680. doi: 10.1186/s12864-017-4029-3

Yang, L., Bin, Z., Hui, S., Rong, L., You, B., Wu, P., et al. (2019). The role of CDR1as in proliferation and differentiation of human umbilical cord-derived mesenchymal stem cells. Stem Cells Int. 2019:2316834. doi: 10.1155/2019/ 2316834

Yousefi, F., Shabaninejad, Z., Vakili, S., Derakhshan, M., Movahedpour, A., Dabiri, H., et al. (2020). TGF- $\beta$ and WNT signaling pathways in cardiac fibrosis: non-coding RNAs come into focus. Cell Commun. Signal 18:87. doi: 10.1186/s12964-020-00555-4

Zeng, K., Chen, X., Xu, M., Liu, X., Hu, X., Xu, T., et al. (2018). CircHIPK3 promotes colorectal cancer growth and metastasis by sponging miR-7. Cell Death Dis. 9:417. doi: 10.1038/s41419-018-0454-8

Zhang, J., Chen, S., Yang, J., and Zhao, F. (2020a). Accurate quantification of circular RNAs identifies extensive circular isoform switching events. Nat. Commun. 11:90. doi: 10.1038/s41467-019-13840-9

Zhang, M., Wang, Z., Cheng, Q., Wang, Z., Lv, X., Wang, Z., et al. (2020b). Circular RNA (circRNA) CDYL induces myocardial regeneration by ceRNA after myocardial infarction. Med. Sci. Monit. 26:e923188. doi: 10.12659/ MSM.923188

Zhang, Y., Zhang, X. -O., Chen, T., Xiang, J. -F., Yin, Q. -F., Xing, Y. -H., et al. (2013). Circular intronic long noncoding RNAs. Mol. Cell 51, 792-806. doi: 10.1016/j.molcel.2013.08.017

Zhao, Z., Li, X., Gao, C., Jian, D., Hao, P., Rao, L., et al. (2017). Peripheral blood circular RNA hsa_circ_0124644 can be used as a diagnostic biomarker of coronary artery disease. Sci. Rep. 7:39918. doi: 10.1038/srep39918

Zheng, Q., Bao, C., Guo, W., Li, S., Chen, J., Chen, B., et al. (2016). Circular RNA profiling reveals an abundant circHIPK3 that regulates cell growth by sponging multiple miRNAs. Nat. Commun. 7:11215. doi: 10.1038/ ncomms 11215

Zhong, Q., Huang, J., Wei, J., and Wu, R. (2019). Circular RNA CDRlas sponges miR-7-5p to enhance E2F3 stability and promote the growth of nasopharyngeal carcinoma. Cancer Cell Int. 19:252. doi: 10.1186/s12935019-0959-y 
Zhu, Y., Pan, W., Yang, T., Meng, X., Jiang, Z., Tao, L., et al. (2019). Upregulation of circular RNA CircNFIB attenuates cardiac fibrosis by sponging miR-433. Front. Genet. 10:564. doi: 10.3389/fgene.2019.00564

Conflict of Interest: The authors declare that the research was conducted in the absence of any commercial or financial relationships that could be construed as a potential conflict of interest.
Copyright $\odot 2020$ Mester-Tonczar, Hašimbegović, Spannbauer, Traxler, Kastner, Zlabinger, Einzinger, Pavo, Goliasch and Gyöngyösi. This is an open-access article distributed under the terms of the Creative Commons Attribution License (CC BY). The use, distribution or reproduction in other forums is permitted, provided the original author(s) and the copyright owner(s) are credited and that the original publication in this journal is cited, in accordance with accepted academic practice. No use, distribution or reproduction is permitted which does not comply with these terms. 